\title{
Graft failure of $\lg A$ nephropathy in renal allografts following living donor transplantation: predictive factor analysis of 102 biopsies
}

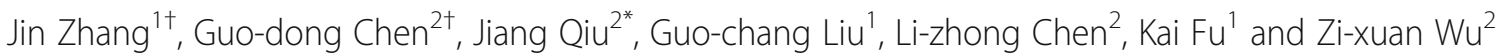

\begin{abstract}
Background: To investigate predictive factors related to graft failure of IgA nephropathy(IgAN) in renal allografts following living donor transplantation.

Methods: We identified a series of 102 biopsies diagnosed as IgAN in renal allografts following living donor transplantation from July 2004 to January 2017 at our center, and assess the predict value of the Lee's classification and the 2009 Oxford classification in IgAN in renal allografts, clinical, ultrasonic and pathological characteristics at biopsy and the outcomes were retrospectively analyzed.

Results: The 5-year graft cumulative survival rate after transplantation was 91.4\%. The 4-year graft cumulative survival rate after biopsy diagnosis of IgAN in renal allografts was $59.6 \%$. The mean time \pm SD to disease was $4.7 \pm$ 3.5 years. The color doppler ultrasound and blood flow imagine showed the echo enhancement, the reduced blood flow distribution, the reduced peak systolic velocity of main renal artery, and the increased resistance index of arcuate renal artery were valuable in evaluating the graft dysfunction. The Cox multivariate analysis revealed that the 24-h urinary protein level (HR 1.6 for $1-\mathrm{g}$ increase, $95 \% \mathrm{Cl} 1.2-2.0)$, estimated glomerular filtration rate (eGFR) (HR 1.0 for $1-\mathrm{mL} / \mathrm{min} / 1.73 \mathrm{~m} \wedge 2$ decline, $95 \% \mathrm{Cl} 1.0-1.1$ ), and mesangial C1q deposition (HR 3.0, 95\%Cl 1.2-7.4) at biopsy were independent predictive factors of graft failure of IgAN in renal allografts.

Conclusions: IgAN in renal allografts occurred frequently within 5 years after transplantation. The risk of graft failure should be taken seriously in patients who exhibit heavy proteinuria and/or a declined eGFR as the initial symptoms; a high lesion grade (grade IV-V of Lee's classification) and/or mesangial C1q deposition may also indicated a poor outcome.
\end{abstract}

Keywords: IgA nephropathy, Graft failure, Living donor transplantation, Biopsy

\section{Background}

The widespread use of effective immunosuppression has markedly reduced the risk of graft failure due to rejection in recent year s[1]. Conversely, de novo or recurrent renal disease has become a major cause of graft dysfunction, which was confirmed in our previous study,[2] and it is the second leading cause of death-censored graft

\footnotetext{
* Correspondence: 502067665@qq.com

†jin Zhang and Guo-dong Chen contributed equally to this work.

${ }^{2}$ Department of Organ Transplant, the First Affiliated Hospital of Sun Yat-sen

University, Guangzhou 510000, China

Full list of author information is available at the end of the article
}

failure and the third leading cause of uncensored graft failur e[3, 4].

IgA nephropathy (IgAN) is the most common recurrent nephropathy after transplantation, especially living donor transplantatio $\mathrm{n}[5,6]$. Some scholar s[5, 7] have suggested that IgAN recurrence is an independent risk factor for graft failure, but few studies have investigated the risk factors of IgAN in renal allografts that are predictive of disease progression and the outcomes compared to native IgA N $[8,9]$. Therefore, the prognosis of IgAN in renal allografts remains controversia $1[7,10,11]$. The aim of this study was to investigate the predictive factors related to

(c) The Author(s). 2019 Open Access This article is distributed under the terms of the Creative Commons Attribution 4.0 International License (http://creativecommons.org/licenses/by/4.0/), which permits unrestricted use, distribution, and 
graft failure of IgAN in renal allografts following living donor transplantation.

\section{Methods \\ Sample collection}

In a total of 637 living donor transplantations in the First Affiliated Hospital of Sun Yat-sen University (Guangzhou, China) from July 2004 to January 2017, we identified a series of 102 patients with a diagnosis of IgAN in renal allografts proven by biopsy, including 93 indication biopsies and 9 protocol biopsies, repeated biopsies with a same diagnosis were excluded. The series consisted of 72 males and 30 females with a mean age $\pm S D$ of $35.5 \pm 9.1$ years. Sixty-seven patients have been proven primary IgAN by biopsy for native renal in this series.

A follow-up was performed in the First Affiliated Hospital of Sun Yat-sen University (Guangzhou, China) and Guangzhou Women and Children's Medical Center (Guangzhou, China). Routine blood and urine examinations, liver and renal function tests, and therapeutic drug monitoring were conducted prior to biopsy for all patients. The standard maintenance therapy included a calcineurin inhibitor (CNI) (Tacrolimus was initiated at $0.05 \mathrm{mg} / \mathrm{kg} \mathrm{q} 12 \mathrm{~h}$ or Cyclosporine A was initiated at $2-5$ $\mathrm{mg} / \mathrm{kg} \mathrm{q} 12 \mathrm{~h}$, the dosage adjusted according to the blood concentrations) in combination with mycophenolate mofetil (MMF) (Mycophenolate Sodium was initiated at $360 \mathrm{mg}$ q12h) and corticosteroids. Oral prednisone was initiated at $30 \mathrm{mg}$ (qd) and gradually reduced to 5-10 mg (qd) over a 4-week period. Table 1 lists other clinical characteristics and the therapeutic regimen after disease occurrence.

The Regional Ethics Committee of our center approved this study, and all patients signed informed consent forms.

\section{Biopsy and diagnosis of IgAN in renal allografts}

The following indications for biopsy were combination of doctors' experience and the 2009 KDIGO Clinical Practice Guideline for the Care of Kidney Transplant Recipient $s$ [12] were used: 1 ) continuous anuria or oliguria $(<400 \mathrm{ml} / 24 \mathrm{~h}) ; 2)$ durative hematuria or proteinuria (positive in urine examinations for more than 1 month); 3) continuous increase in serum creatinine $(\mathrm{sCr})(>30 \%$ of the baseline) or a concentration above the normal level; 4) B-scan ultrasonography showing an abnormal blood flow peak systolic velocity (Vs) and resistance index (RI); and 5) panel-reactive antibody (PRA) level > $0 \%$ or the presence of donor-specific antibodies (DSAs).

Ninety-three patients with indications were suggested accept biopsy in 1 month, most of them (80.6\%) underwent a timely biopsy, the mean \pm SD waiting time for biopsy was $0.7 \pm 1.2$ months.
Nine patients with high risk factors for recurrence, such as a family history or primary IgAN diagnosed by native renal biopsy, underwent protocol biopsies at 6 months and at 1, 2, 5 and 10 years.

An ultrasonography-guided needle biopsy was performed using an 18-gauge needle (Bard). Each sample included at least 6 glomeruli visible by light microscopy. Immunofluorescence analyses were performed for all biopsies, and the IgA, IgG, IgM, C3, C1q and C4D levels were graded by two senior pathologists in an independent and blinded fashion. A diagnosis of IgAN in renal allografts based on IgA-positivity due to immunofluorescence staining deposition in the mesangial area (Fig. 1), which caused by lupus nephritis (LN) or other renal graft diseases was exclude $d[13]$. The classification were determined based on Lee's and the 2009 Oxford classifications.

The ultrasonic data at the time of the biopsy diagnosis were recorded simultaneously, included cortical thickness, echo enhancement, peak systolic velocity (Vs) and RI in each renal artery.

\section{Statistical analysis}

According to $2002 \mathrm{~K} / \mathrm{DOQI}$ clinical practice guidelines for chronic kidney disease,[14] we defined the graft failure as the endpoint, which means that an estimated glomerular filtration rate (eGFR) less than $30 \mathrm{~mL} / \mathrm{min} /$ $1.73 \mathrm{~m}^{\wedge} 2$ (estimated by CKD-EPI Equation), without recovery for more than 3 months or returned to dialysis immediately.

We analyzed the time to disease recurrence, the 5-year graft cumulative survival rate after transplantation and the 4-year graft cumulative survival rate after biopsy diagnosis. Because the aim of this study was to investigate the predictive factors related to graft failure of IgAN in renal allografts rather than recurrence itself, we defined the initiation of the follow-up time from the day of biopsy diagnosis instead of the day of transplantation.

The urinary red blood cell (U-RBC) count, 24-h urinary protein level, serum albumin (ALB), eGFR, degree of histopathological injury, ultrasonic and other clinical characteristics at the time of the biopsy diagnosis were recorded. Based on Lee's classifications, ultrasonic and blood flow index such as cortical thickness, echo enhancement, Vs and RI were compared in different degree of histopathological injury. Variables were compared using the Mann-Whitney $\mathrm{U}$ test or Chi-square test, and $p$ values lower than 0.05 were considered significant. Correlation analyses were performed among abovementioned characteristics, Pearson's and Spearman's correlation coefficient were used for continuous and categorical variables respectively.

Univariate Cox proportional hazards models were used to investigate the significance of predictive factors related to graft failure, and significant factors were tested 
Table 1 Baseline characteristics of the recipients and donors

\begin{tabular}{|c|c|c|c|}
\hline Variables & $\begin{array}{l}\text { Graft survival } \\
(n=76)\end{array}$ & $\begin{array}{l}\text { Graft failure } \\
(n=26)\end{array}$ & $p$-value \\
\hline Recipient age (years) & $34.1 \pm 8.5$ & $39.3 \pm 9.7$ & 0.05 \\
\hline Recipient sex, f/m (\%) & $18 / 58(24 / 76)$ & $9 / 17(35 / 65)$ & 0.27 \\
\hline Recipient race, East Asian (\%) & $76(100)$ & $26(100)$ & - \\
\hline Diabetes (\%) & $14(18.4)$ & $5(19.2)$ & 0.93 \\
\hline $\mathrm{SBP}(\mathrm{mmHg})$ & $146.3 \pm 17.6$ & $149.5 \pm 19.4$ & 0.92 \\
\hline $\mathrm{DBP}(\mathrm{mmHg})$ & $74.4 \pm 9.5$ & $75.9 \pm 10.5$ & 0.50 \\
\hline Acute rejection ${ }^{\mathrm{a}}(\%)$ & $7(9.2)$ & $4(15.4)$ & 0.38 \\
\hline Delayed graft function (\%) & $4(5.2)$ & $2(7.7)$ & 0.65 \\
\hline Anti-HLA antibodies ${ }^{\mathrm{b}}(\%)$ & $13(17.1)$ & $4(15.4)$ & 0.84 \\
\hline Class I (\%) & $5(6.6)$ & $3(11.5)$ & 0.42 \\
\hline Class II (\%) & $13(17.1)$ & $2(7.7)$ & 0.24 \\
\hline HLA mismatches ( $A, B$, or DR) & $2.1 \pm 1.1$ & $1.7 \pm 1.0$ & 0.77 \\
\hline \multicolumn{4}{|l|}{ Therapeutic regimen } \\
\hline Intravenous methylprednisolone pulses ${ }^{\complement}(\%)$ & $21(27.6)$ & $7(26.9)$ & 0.94 \\
\hline Tonsillectomy (\%) & $10(13.1)$ & $6(23.1)$ & 0.23 \\
\hline ACEls used (\%) & $59(77.6)$ & $23(88.5)$ & 0.23 \\
\hline Disease time $^{d}$ (years) & $4.0 \pm 3.4$ & $5.3 \pm 3.7$ & 0.62 \\
\hline Waiting time $\mathrm{e}^{\mathrm{e}}$ (months) & $0.8 \pm 1.3$ & $0.4 \pm 0.6$ & 0.14 \\
\hline 24-h urinary protein (g/24 h) & $0.3 \pm 0.2$ & $0.4 \pm 0.3$ & 0.10 \\
\hline Urinary RBC count (u/ml) & $1.5 \pm 1.8$ & $2.0 \pm 2.5$ & 0.22 \\
\hline Cholesterol (mmol/L) & $5.3 \pm 1.5$ & $5.7 \pm 2.5$ & 0.36 \\
\hline LDL (mmol/L) & $3.4 \pm 1.1$ & $3.8 \pm 1.6$ & 0.16 \\
\hline $\mathrm{ALB}(\mathrm{g} / \mathrm{L})$ & $42.0 \pm 4.4$ & $40.8 \pm 4.4$ & 0.24 \\
\hline $\mathrm{eGFR}^{\mathrm{f}}\left(\mathrm{mL} / \mathrm{min} / 1.73 \mathrm{~m}^{\wedge} 2\right)^{\mathrm{g}}$ & $75.2 \pm 21.8$ & $67.9 \pm 20.7$ & 0.14 \\
\hline Donor age (years) & $50.4 \pm 8.6$ & $49.5 \pm 8.4$ & 0.77 \\
\hline Donor sex f/m (\%) & $53 / 23(69 / 31)$ & $14 / 12(54 / 46)$ & 0.14 \\
\hline Donor race, East Asian (\%) & $76(100)$ & $26(100)$ & - \\
\hline
\end{tabular}

SBP Systolic blood pressure, DBP Diastolic blood pressure, HLA Human leukocyte antigen, $A C E l s$ Angiotensin-converting enzyme inhibitors, RBC Red blood cell, $L D L$ Low-density lipoprotein, ALB Albumin, eGFR Estimated glomerular filtration rate

a patients with a history of acute rejection after transplantation were included if they were cured when diagnosed as IgAN

bincluding donor-specific antibodies and non-donor specific antibodies

cthree intravenous methylprednisolone pulses at the beginning of recurrence, but routine anti-rejection treatments after transplantation were excluded

dime from transplantation to the onset of initial symptoms

time from the onset of the initial symptoms to biopsy

fbaseline eGFR after transplantation

${ }^{g}$ the volume of urine filtrated by glomeruli every $1 \mathrm{~min}$ in a body surface area of 1.73 square metre

in the multivariate analysis. Kaplan-Meier estimates were used to create graft survival curves, and comparisons were performed using the log-rank test.

The data were analyzed using the IBM SPSS Statistics software version 22.0.

\section{Results}

Ninety-three patients who were diagnosed with IgAN in renal allografts underwent a biopsy for initial symptoms. Proteinuria occurred in 72 patients (70.6\%), hematuria occurred in 65 patients (63.7\%), hypoproteinemia occurred in 18 patients (17.6\%), and increased $\mathrm{sCr}(>30 \%$ of the baseline) occurred in 28 patients (27.4\%). Nine patients $(8.8 \%)$ did not have abnormalities and were diagnosed using the protocol biopsy.

Graft failure occurred in 26 patients. The 5-year graft cumulative survival rate was $91.4 \%$ after transplantation (mean \pm SD follow up, $7.6 \pm 3.7$ years), and the 4-year graft cumulative survival rate after diagnosis of IgAN by biopsy was $59.6 \%$ (the 5-year graft cumulative survival rate after diagnosis was not obtained, because the mean \pm SD follow up was $3.4 \pm 1.9$ years). The mean time \pm SD to occurrence was $4.7 \pm 3.5$ years. Sixty-four cases (62.7\%) occurred within 5 years after 

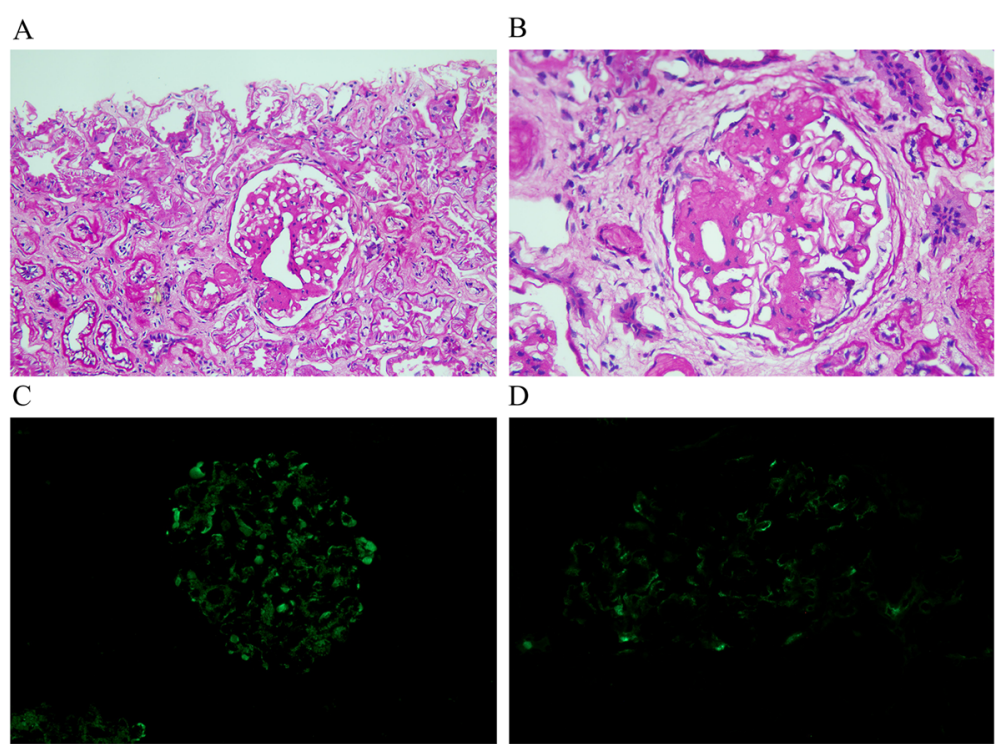

Fig. 1 Pathological characters of IgAN in renal allografts (Lee's IV, Oxford M1E1S1T1). a Glomerular mesangial proliferation and endocapillary hypercellularity, with part of the renal tubular atrophy. (PAS, $\times 200)$. b The basement membrane fragmented and absent, segment glomerular sclerosis and cellular/fibrocellular crescent formation. (PAS, $\times 400$ ). $\mathbf{c}$ The positivity of mesangial IgA deposition. (Immunofluorescence staining). $\mathbf{d}$ The positivity of mesangial C1q deposition. (Immunofluorescence staining)

transplantation, and 38 cases (37.2\%) occurred after more than 5 years (Fig. 2).

The color doppler ultrasound and blood flow imagine showed the echo enhancement $(p<0.01)$, the reduced blood flow distribution $(p=0.02)$, the reduced Vs of main renal artery (MRA) $(p=0.04)$, and the increased RI of arcuate renal artery (ARA) $(p=0.04)$ were valuable in diagnosing and evaluating the graft dysfunction (Table 2). There was a positive correlation between Lee's classifications and echo enhancement (Spearman's correlation

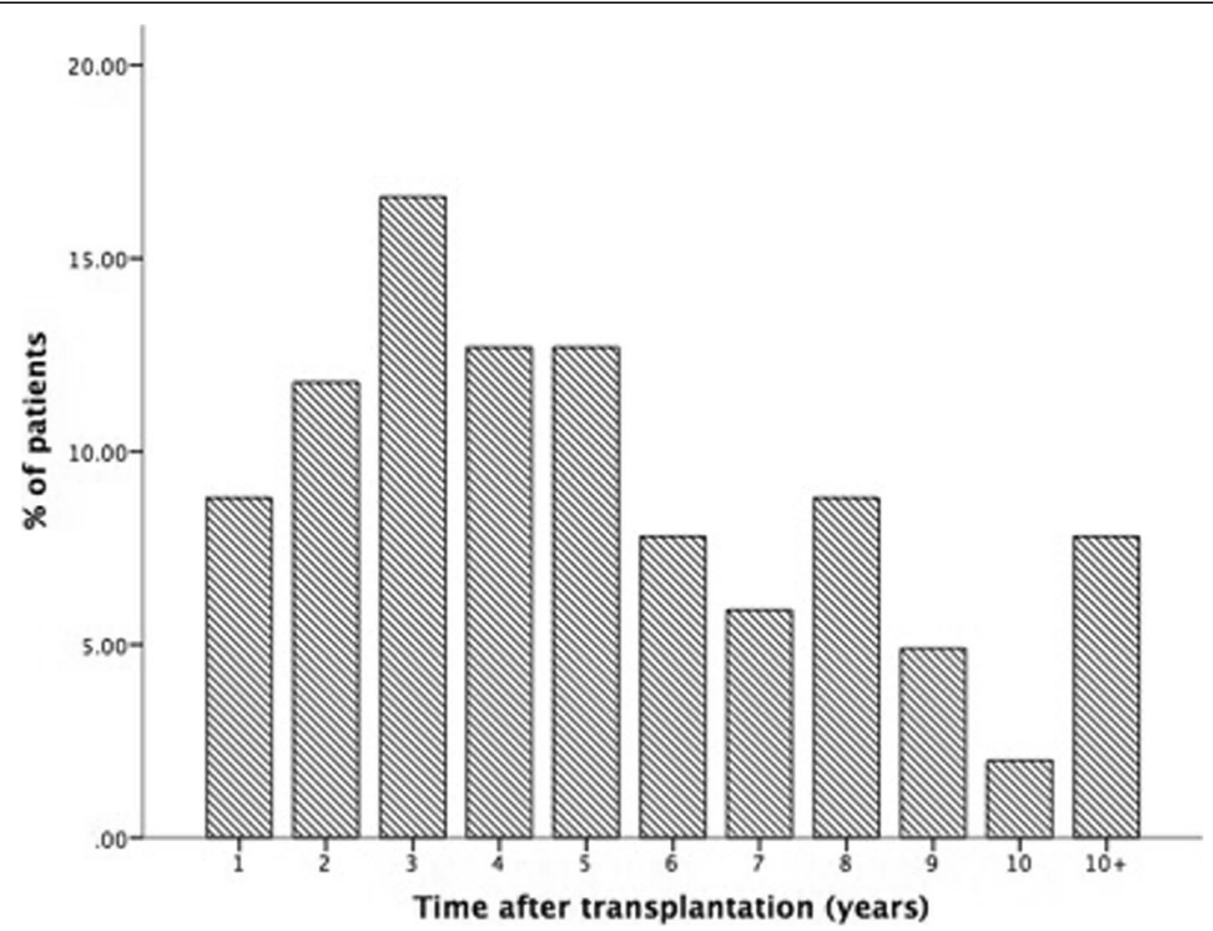

Fig. 2 Duration from transplantation to IgAN in renal allografts 
Table 2 Color doppler ultrasound and blood flow index (based on Lee's classification)

\begin{tabular}{|c|c|c|c|c|c|c|}
\hline \multirow[t]{2}{*}{ Variable } & \multicolumn{5}{|c|}{ Lee's classification (I-V) $\left(n=75^{\mathrm{a}}\right)$} & \multirow{2}{*}{$\begin{array}{l}p \\
\text { value }\end{array}$} \\
\hline & $\begin{array}{l}(n=19) \\
(n)\end{array}$ & $\begin{array}{l}\| \\
(n=13)\end{array}$ & $\begin{array}{l}\text { III } \\
(n=26)\end{array}$ & $\begin{array}{l}\text { IV } \\
(n=14)\end{array}$ & $\begin{array}{l}V \\
(n=3)\end{array}$ & \\
\hline${\text { Cortical thickness1 } 1^{\mathrm{ab}}(\mathrm{cm})}$ & $1.5 \pm 0.3$ & $1.5 \pm 0.2$ & $1.5 \pm 0.2$ & $1.6 \pm 0.3$ & $1.5 \pm 0.2$ & 0.40 \\
\hline 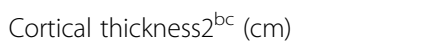 & $1.7 \pm 0.6$ & $1.5 \pm 0.3$ & $1.5 \pm 0.3$ & $1.5 \pm 0.3$ & $1.3 \pm 0.2$ & 0.59 \\
\hline Change in cortical thickness ${ }^{\mathrm{cd}}(\mathrm{cm})$ & $-0.1 \pm 0.6$ & $0.1 \pm 0.4$ & $0 \pm 0.2$ & $0.1 \pm 0.3$ & $0.2 \pm 0.1$ & 0.23 \\
\hline Echo enhancement (\%) & $2(10.5)$ & $1(7.7)$ & $2(7.7)$ & $8(57.1)$ & $2(66.7)$ & $<0.01$ \\
\hline Blood flow distribution1 (I-V) & $4.9 \pm 0.3$ & $4.8 \pm 0.4$ & $4.9 \pm 0.3$ & $4.9 \pm 0.4$ & $5.0 \pm 0$ & 0.96 \\
\hline Blood flow distribution2 (I-V) & $4.8 \pm 0.4$ & $4.6 \pm 0.6$ & $4.7 \pm 0.5$ & $4.4 \pm 0.7$ & $3.7 \pm 1.1$ & 0.06 \\
\hline Change in blood flow distribution (I-V) & $0.5 \pm 0.2$ & $0.2 \pm 0.4$ & $0.1 \pm 0.4$ & $0.5 \pm 0.8$ & $1.3 \pm 1.1$ & 0.02 \\
\hline \multicolumn{7}{|l|}{ MRA } \\
\hline Vs1 $(\mathrm{cm} / \mathrm{s})$ & $91.7 \pm 20.0$ & $109.4 \pm 46.6$ & $102.1 \pm 26.8$ & $100.1 \pm 30.7$ & $133.6 \pm 45.7$ & 0.31 \\
\hline Vs2 (cm/s) & $104.6 \pm 54.1$ & $114.8 \pm 48.3$ & $117.2 \pm 49.1$ & $82.2 \pm 32.1$ & $95.4 \pm 35.2$ & 0.20 \\
\hline Vs1- Vs2 (cm/s) & $-12.9 \pm 61.1$ & $-5.4 \pm 26.7$ & $-15.1 \pm 51.4$ & $17.9 \pm 24.6$ & $38.3 \pm 10.5$ & 0.04 \\
\hline Rl1 & $0.6 \pm 0.1$ & $0.7 \pm 0.1$ & $0.7 \pm 0.1$ & $0.7 \pm 0.1$ & $0.6 \pm 0$ & 0.80 \\
\hline $\mathrm{R} / 2$ & $0.7 \pm 0.1$ & $0.7 \pm 0.1$ & $0.7 \pm 0.1$ & $0.7 \pm 0.1$ & $0.7 \pm 0$ & 0.36 \\
\hline RI1/RI2 & $1.0 \pm 0.2$ & $1.0 \pm 0.1$ & $1.0 \pm 0.1$ & $0.9 \pm 0.1$ & $0.9 \pm 0.1$ & 0.15 \\
\hline \multicolumn{7}{|l|}{ SRA } \\
\hline Vs1 (cm/s) & $54.2 \pm 17.4$ & $59.0 \pm 18.1$ & $55.3 \pm 14.7$ & $61.6 \pm 19.0$ & $81.3 \pm 2.1$ & 0.14 \\
\hline Vs2 (cm/s) & $53.1 \pm 15.1$ & $60.6 \pm 18.8$ & $56.6 \pm 13.0$ & $52.4 \pm 21.4$ & $45.5 \pm 3.5$ & 0.38 \\
\hline Vs1- Vs2 (cm/s) & $1.1 \pm 20.8$ & $-1.6 \pm 12.6$ & $-1.3 \pm 17.7$ & $9.1 \pm 23.6$ & $35.8 \pm 5.3$ & 0.05 \\
\hline RI1 & $0.6 \pm 0.1$ & $0.6 \pm 0.1$ & $0.6 \pm 0.1$ & $0.6 \pm 0.1$ & $0.6 \pm 0.1$ & 0.66 \\
\hline $\mathrm{R} / 2$ & $0.6 \pm 0.1$ & $0.6 \pm 0$ & $0.6 \pm 0.1$ & $0.7 \pm 0.1$ & $0.7 \pm 0.1$ & 0.15 \\
\hline RI1/RI2 & $1.0 \pm 0.1$ & $1.0 \pm 0.1$ & $1.0 \pm 0.1$ & $0.9 \pm 0.1$ & $0.9 \pm 0.1$ & 0.18 \\
\hline \multicolumn{7}{|l|}{ IRA } \\
\hline Vs1 (cm/s) & $29.4 \pm 8.0$ & $34.5 \pm 9.1$ & $31.2 \pm 8.0$ & $34.2 \pm 9.1$ & $37.4 \pm 10.7$ & 0.26 \\
\hline Vs2 (cm/s) & $31.2 \pm 10.3$ & $34.5 \pm 7.5$ & $32.7 \pm 8.5$ & $28.6 \pm 10.9$ & $28.5 \pm 10.5$ & 0.42 \\
\hline Vs1-Vs2 (cm/s) & $-1.8 \pm 11.4$ & $0 \pm 9.3$ & $-1.5 \pm 10.2$ & $5.6 \pm 9.3$ & $8.8 \pm 8.3$ & 0.10 \\
\hline RI1 & $0.6 \pm 0.1$ & $0.6 \pm 0.1$ & $0.6 \pm 0.1$ & $0.6 \pm 0$ & $0.6 \pm 0$ & 0.92 \\
\hline $\mathrm{R} / 2$ & $0.6 \pm 0.1$ & $0.6 \pm 0.1$ & $0.6 \pm 0.1$ & $0.6 \pm 0.1$ & $0.7 \pm 0$ & 0.11 \\
\hline RI1/RI2 & $1.0 \pm 0.2$ & $1.0 \pm 0.1$ & $1.0 \pm 0.1$ & $0.9 \pm 0.1$ & $0.9 \pm 0$ & 0.08 \\
\hline \multicolumn{7}{|l|}{ ARA } \\
\hline Vs1 (cm/s) & $20.8 \pm 6.7$ & $21.6 \pm 4.4$ & $19.7 \pm 5.3$ & $25.3 \pm 7.2$ & $27.4 \pm 6.2$ & 0.07 \\
\hline Vs2 (cm/s) & $20.2 \pm 8.0$ & $22.1 \pm 8.2$ & $20.9 \pm 9.5$ & $18.9 \pm 8.0$ & $19.6 \pm 4.5$ & 0.79 \\
\hline Vs1- Vs2 (cm/s) & $0.6 \pm 7.3$ & $-0.5 \pm 9.3$ & $-1.2 \pm 13.0$ & $6.4 \pm 9.1$ & $7.8 \pm 1.8$ & 0.07 \\
\hline Rl1 & $0.6 \pm 0$ & $0.6 \pm 0$ & $0.6 \pm 0.1$ & $0.6 \pm 0$ & $0.6 \pm 0$ & 0.85 \\
\hline RI2 & $0.6 \pm 0.1$ & $0.6 \pm 0.1$ & $0.6 \pm 0.1$ & $0.6 \pm 0.1$ & $0.7 \pm 0.1$ & 0.04 \\
\hline RI1/RI2 & $1.0 \pm 0.1$ & $1.0 \pm 0.1$ & $1.1 \pm 0.2$ & $1.0 \pm 0.1$ & $0.9 \pm 0$ & 0.04 \\
\hline
\end{tabular}

ain total series, 27 cases without complete ultrasonic data

${ }^{b}$ the baseline level at the time when patients had the lowest $\mathrm{s} C$ level after transplantation, the same definition for following index the level at the time of biospy, the same definition for following index

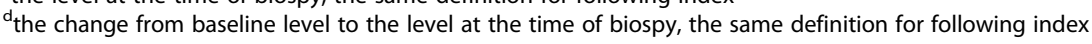

coefficient $=0.4, p<0.01$ ), the reduced blood flow distribution (Spearman's correlation coefficient $=0.3, p<0.01$ ), the reduced Vs of MRA (Spearman's correlation coefficient $=0.3, p=0.03$ ), and the increased RI of ARA (Spearman's correlation coefficient $=0.3, p=0.03$ ).
The univariate analysis revealed that age $(p=0.05)$, time to disease occurrence $(p=0.05)$, the 24-h urinary protein level $(p<0.01)$, eGFR $(p<0.01)$, hypoproteinemia $(\mathrm{ALB}<35.0 \mathrm{~g} / \mathrm{L})(p<0.01)$, Lee's classification $(p=$ $0.02)$, mesangial C1q deposition $(p<0.01)$ at the time of 
biopsy were predictive of graft failure. We found significant correlations between the abovementioned characteristics. For instance, the time to disease occurrence was directly correlated with age (Pearson's correlation coefficient $=0.4, p<0.01$ ), Lee's classification (Spearman's correlation coefficient $=0.3, p<0.01$ ), and mesangial C1q deposition (Spearman's correlation coefficient $=0.2, p=$ 0.02 ). The 24-h urinary protein level was directly correlated with hypoproteinemia (Spearman's correlation coefficient $=0.4, p<0.01$ ). The multivariate analysis demonstrated that the 24-h urinary protein level, eGFR and mesangial $\mathrm{C} 1 \mathrm{q}$ deposition at the time of the biopsy were independent predictive factors related to graft failure. The hazard ratios (HRs) were 1.6 for 1 -g increased 24-h urinary protein $(95 \% \mathrm{CI} 1.2-2.0), 1.0$ for $1-\mathrm{mL} / \mathrm{min} / 1.73 \mathrm{~m}^{\wedge} 2$ declined eGFR (95\%CI 1.0-1.1), 3.0 for mesangial C1q deposition (95\%CI 1.2-7.4) (Table 3).

Although some characteristics were not independent predictive factors of graft failure in our study, the log-rank test revealed a significant difference between the KaplanMeier curves of Lee's classification $(p<0.01)$ (Table 4$)$. Figure 3 shows the significant differences in the 24-h urinary protein level $(p<0.01)$ and other factors.

\section{Discussion}

Due to differences in diagnostic criteria, discrepancies have been reported in recurrence rates, with a range from 9 to $61 \%[5]$. Family history of IgAN is a known factor of its recurrence,[15] combined with a review of the Australia-New Zealand registry,[6] IgAN patients accepted low HLA-mismatched living donor transplantation with higher risk of recurrence, thus the family history in cases who accepted related donors should be given more attention.

More and more recent studies confirmed that IgAN in renal allografts had impact on long-term graft survival, $[9,10,16]$ which were inconsistent with early research suggested that it exhibited slow progression and benign outcomes,[11] especially in patients with asymptomatic hematuria and/or proteinuria. Floege et al .[17] reported that $\sim 5 \%$ of patients lost their grafts due to occurrence at 5 years after transplantation, and our study demonstrated a similar result. However, the 4-year graft cumulative survival rate was only $59.6 \%$ after the IgAN diagnosis, which showed a worse outcome in our study.

We found that IgAN occurred frequently within 5 years postoperatively and then declined sharply in subsequent years, which was consistent with a previous repor $\mathrm{t}[18]$. Few patients diagnosed with IgAN in renal allografts showed a remarkable increased $\mathrm{sCr}$ level when accepted biopsy,[2] as reported in previous study on IgAN in renal allografts, it had little impact on graft function in the early years after transplantation,[19] but Ortiz et al .20] found that the histopathological injury was not accompanied by abnormalities in the urinalysis in half of early recurrent IgAN patients. Therefore, the disease progression could be concealed, a protocol biopsy was recommended to prevent missed diagnoses, especially in clinically silent patients.

An increased sCr level or declined eGFR as the initial symptoms was generally associated with a high risk of graft failure. This result was confirmed in a previous study on native IgA N[21]. Another independent predictive factor related to renal failure in native and graft IgAN was the 24-h urinary protein level, which was confirmed in our and other recent studie s[22, 23]. This risk was obviously increased when proceeding to hypoproteinemia. Hematuria is a typical clinical symptom of IgAN, and one prognostic factor study in Chinese patients concluded that hematuria was a useful marker for patients who were at high risk for disease progression of native IgA N[24]. However, no correlation was found between hematuria and graft failure in our study, possibly for the following reasons: 1) IgAN in renal allografts is not the only cause of hematuria following transplantation, which is different than native IgAN, and drug toxicity damage, infection, use of a Double-J catheter and other factors may induce hematuria in these patients; 2) some patients with initial asymptomatic hematuria did not accept a biopsy in a timely manner; and 3) only 26 cases of graft failure were observed in this series. Thus, more samples and a longer-term follow-up are needed to test the predictive value of hematuria.

The color doppler ultrasound is helpful to evaluate the progress of renal graft diseases, the change in cortical thickness, echo enhancement and the reduced blood flow distribution are typical features of graft dysfunction, like echo enhancement denotes glomerulosclerosis and interstitial fibrosis, which showed in our results. The results indicate that patients with severe histopathological injury (grade IV-V of Lee's classification) may show lower Vs and higher RI in blood flow than mild or moderate (grade I-III), it coincide partly with early research, [25] which is concluded that it can be diagnosed as renal disease when RI $>0.65$ in interlobar renal artery (IRA), but we found that the RI fluctuated between 0.6 and 0.7 in most cases of IgAN in renal allografts, even if they had a good graft function, moreover, the blood flow in MRA and ARA had more significant changes than it in IRA. These differences may be concerned with the baseline level influenced by donor factors, in addition, handlers' skills and experience are important influence factors.

Lee's classification and the Oxford classification are useful prognostic indicators of disease progression and clinical outcomes of native IgAN, but their utility in IgAN in after transplantation remains unknown. Lee's classification includes criteria for histopathological grading, such as glomerular sclerosis, crescent formation, 
Table 3 Predictive factors related to graft failure of IgAN in renal allografts

\begin{tabular}{|c|c|c|c|c|}
\hline \multirow[t]{2}{*}{ Predictive factors } & \multicolumn{2}{|l|}{ Univariate } & \multicolumn{2}{|l|}{ Multivariate } \\
\hline & $\mathrm{HR}(95 \% \mathrm{Cl})$ & $p$ value & $\mathrm{HR}(95 \% \mathrm{Cl})$ & $p$ value \\
\hline Age (years) & $1.0(1.0-1.1)$ & 0.03 & - & 0.38 \\
\hline Disease time (years) & $1.1(1.0-1.3)$ & 0.03 & - & 0.38 \\
\hline Waiting time ${ }^{a}$ (months) & $0.7(0.4-1.2)$ & 0.20 & - & - \\
\hline $\mathrm{SBP}(\mathrm{mmHg})$ & $1.0(0.9-1.0)$ & 0.90 & - & - \\
\hline $\mathrm{DBP}(\mathrm{mmHg})$ & $1.0(1.0-1.1)$ & 0.55 & - & - \\
\hline 24-h urinary protein ( $\mathrm{g} / 24 \mathrm{~h})$ & $1.6(1.3-2.0)$ & $<0.01$ & $1.6(1.2-2.0)$ & $<0.01$ \\
\hline \multicolumn{5}{|l|}{ Hematuria $(-$ to +++$)$} \\
\hline- & 1.0 & 0.80 & - & - \\
\hline+ & $0.6(0.2-1.7)$ & 0.38 & - & - \\
\hline++ & $0.7(0.2-1.9)$ & 0.49 & - & - \\
\hline+++ & $0.7(0.1-3.1)$ & 0.63 & - & - \\
\hline Urinary RBC count (u/ml) & $1.0(0.9-1.0)$ & 0.68 & - & - \\
\hline Cholesterol (mmol/L) & $1.0(0.8-1.2)$ & 0.79 & - & - \\
\hline $\mathrm{LDL}(\mathrm{mmol} / \mathrm{L})$ & $1.1(0.8-1.4)$ & 0.57 & - & - \\
\hline$A L B(g / L)$ & $0.9(0.8-0.9)$ & $<0.01$ & - & 0.50 \\
\hline $\mathrm{eGFR}^{\mathrm{b}}\left(\mathrm{mL} / \mathrm{min} / 1.73 \mathrm{~m}^{\wedge} 2\right)^{c}$ & $1.0(1.0-1.1)$ & $<0.01$ & $1.0(1.0-1.1)$ & $<0.01$ \\
\hline \multicolumn{5}{|l|}{ Therapeutic regimen } \\
\hline MPP(\%) & $2.2(0.9-5.5)$ & 0.09 & - & - \\
\hline Tonsillectomy (\%) & $0.8(0.3-2.0)$ & 0.69 & - & - \\
\hline ACEls used (\%) & $1.9(0.6-6.2)$ & 0.31 & - & - \\
\hline \multicolumn{5}{|l|}{ Lee's classification (I-V) } \\
\hline । & 1.0 & 0.02 & - & 0.52 \\
\hline$\|$ & $1.2(0.3-4.9)$ & 0.83 & - & 0.44 \\
\hline III & $1.4(0.4-4.5)$ & 0.54 & - & 0.91 \\
\hline IV & $4.2(1.4-13.1)$ & 0.01 & - & 0.97 \\
\hline V & $5.6(1.3-23.6)$ & 0.02 & - & 0.09 \\
\hline \multicolumn{5}{|l|}{ Oxford classification (MEST) } \\
\hline MO & 1.0 & - & - & - \\
\hline M1 & $1.5(0.2-11.1)$ & 0.69 & - & - \\
\hline EO & 1.0 & - & - & - \\
\hline E1 & $2.1(0.9-4.5)$ & 0.63 & - & - \\
\hline so & 1.0 & - & - & - \\
\hline S1 & $1.1(0.4-2.8)$ & 0.88 & - & - \\
\hline TO & 1.0 & - & - & - \\
\hline $\mathrm{T} 1-2$ & $3.0(0.7-12.7)$ & 0.14 & - & - \\
\hline \multicolumn{5}{|l|}{ Immunofluorescence staining } \\
\hline $\lg A+$ & - & - & - & - \\
\hline $\lg G+$ & $1.4(0.5-4.0)$ & 0.49 & - & - \\
\hline $\lg M+$ & $2.5(0.9-6.7)$ & 0.26 & - & - \\
\hline $\mathrm{C} 3+$ & $0.1(0.4-2.2)$ & 0.85 & - & - \\
\hline $\mathrm{C} 1 \mathrm{q}+$ & $4.0(1.7-9.3)$ & $<0.01$ & $3.0(1.2-7.4)$ & 0.02 \\
\hline$C 4 d+$ & $0.4(0.1-2.8)$ & 0.34 & - & - \\
\hline
\end{tabular}


Table 3 Predictive factors related to graft failure of IgAN in renal allografts (Continued)

\begin{tabular}{lllll}
\hline Predictive factors & Univariate & & Multivariate \\
& HR $(95 \% \mathrm{Cl})$ & $p$ value & & HR $(95 \% \mathrm{Cl})$ \\
\hline Comorbidity & $1.5(0.5-4.4)$ & 0.45 & - \\
Acute rejection & $6.7(0.8-53.1)$ & 0.07 & - \\
Chronic rejection & $2.3(0.5-9.9)$ & 0.25 & - \\
CNI toxicity & - & 0.69 & - \\
BKVAN & - & & - \\
\hline
\end{tabular}

HR Hazard ratio, CI Confidence interval, SBP Systolic blood pressure, DBP Diastolic blood pressure, RBC Red blood cell, $L D L$ Low-density lipoprotein, $A L B$ Ibumin; eGFR Estimated glomerular filtration rate, MPP Methylprednisolone pulse, ACEls Angiotensin-converting enzyme inhibitors, CNI Calcineurin inhibitor, BKVAN BK virus-associated nephropathy

athe time from onset of initial symptoms to biopsy

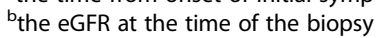

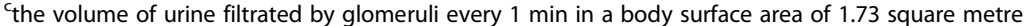

interstitial inflammation and tubular atrophy, which were established based on and considered suitable for East Asian patients. Patients with mild or moderate (grade I-III) lesions have been reported to have a benign outcome, whereas patients with grade IV or V lesions develop end-stage renal failure,[26] which is completely consistent with our results. Thus, Lee's classification exhibits the same utility for prediction of the outcome of IgAN in renal allografts. The 2009 Oxford classification includes the following histological components: mesangial (M) and endocapillary (E) hypercellularity, segmental sclerosis (S) and interstitial fibrosis/tubular atrophy $(\mathrm{T})$. This classification provided a clear definition and accurate criteria for the various pathological lesions of IgAN. It is widely used, and its utility has been validated in many studie s[27, 28]. However, the advantages of this classification system for the clinical prediction of IgAN

Table 4 The 4-year graft cumulative survival rate after biopsy diagnosis of different predictive factors

\begin{tabular}{|c|c|c|c|c|c|}
\hline \multirow{2}{*}{$\begin{array}{l}\text { Predictive } \\
\text { factor }\end{array}$} & \multicolumn{5}{|c|}{ Graft cumulative survival rate after biopsy diagnosis } \\
\hline & 1-year & 2-year & 3-year & 4-year & Log-rank $p$ \\
\hline \multicolumn{6}{|c|}{ 24-h urinary protein ( $\mathrm{g} / 24 \mathrm{~h})$} \\
\hline$<1(n=68)$ & $98.5 \%$ & $85.0 \%$ & $82.1 \%$ & $74.3 \%$ & \\
\hline $1-2(n=20)$ & $95.0 \%$ & $90.0 \%$ & $90.0 \%$ & $40.0 \%$ & \\
\hline$>2(n=14)$ & $71.4 \%$ & $40.8 \%$ & $27.2 \%$ & $27.2 \%$ & $<0.01$ \\
\hline \multicolumn{6}{|l|}{ C1q deposition } \\
\hline$-(n=86)$ & $97.7 \%$ & $86.1 \%$ & $82.2 \%$ & $65.2 \%$ & \\
\hline$+(n=16)$ & $73.7 \%$ & $47.8 \%$ & $47.8 \%$ & $23.9 \%$ & $<0.01$ \\
\hline \multicolumn{6}{|c|}{ Lee's classification (I-V) } \\
\hline I $(n=28)$ & $100 \%$ & $84.5 \%$ & $84.5 \%$ & $77.5 \%$ & \\
\hline$\|(n=19)$ & $100 \%$ & $92.3 \%$ & $79.1 \%$ & $59.3 \%$ & \\
\hline III $(n=37)$ & $94.4 \%$ & $83.4 \%$ & $83.4 \%$ & $68.3 \%$ & \\
\hline IV $(n=15)$ & $73.3 \%$ & $58.7 \%$ & $58.7 \%$ & $19.6 \%$ & \\
\hline$V(n=3)$ & $100 \%$ & $66.7 \%$ & $33.3 \%$ & 0 & $<0.01$ \\
\hline
\end{tabular}

in renal allografts was not observed in our study. We considered that immunosuppressive therapy and donor factors might influence the pathological lesions represented by the four parameters in the Oxford classification. One study confirmed that immunosuppressive therapy reduced the predictive value of the pathological parameters in recurrent IgA N[28] compared with native IgAN. A simple "present" or "absent" for these parameters, such as $\mathrm{M}$ and $\mathrm{E}$, cannot precisely reflect the degree of histopathological graft injury. Therefore, these parameters exhibit lower specificity in patients who accept long-term immunosuppressive therapy. The significance of crescent formation was not listed as a prognostic parameter in the 2009 Oxford classification, but in many follow-up validation studie $s[29,30]$ this parameter was considered prognostic, as a result, the Oxford classification was updated to MEST-C (crescent) in 2017 [31]. This study is the first report to assess the predictive value of these two classifications for IgAN in renal allografts, and the advantages and disadvantages of these classifications should be further assessed. We support the use of a combination of the pathological parameters with clinical features at the time of biopsy to provide earlier risk prediction in IgAN.

Sixteen cases with mesangial C1q deposition in this series. Traditionally, the presence of $\mathrm{C} 1 \mathrm{q}$ staining is viewed as a typical pathological lesion of LN rather than IgA N[13]. However, a more recent stud y[32, 33] confirmed that this parameter played a role and occurred in 0 to $45 \%$ of patients with IgAN. Lee et al .[32] concluded that mesangial $\mathrm{C} 1 \mathrm{q}$ deposition in the glomerulus was associated with a poor renal outcome and severe pathological features in native IgAN. We demonstrated that this parameter exhibited a higher predictive value in IgAN after transplantation. The absence of $\mathrm{C} 1 \mathrm{q}$ deposition is a positive predictive sign as a response to steroid pulse therapy and relief of proteinuri a[33]. Therefore, steroid-resistance nephrotic syndrome and a poorer outcome should be 

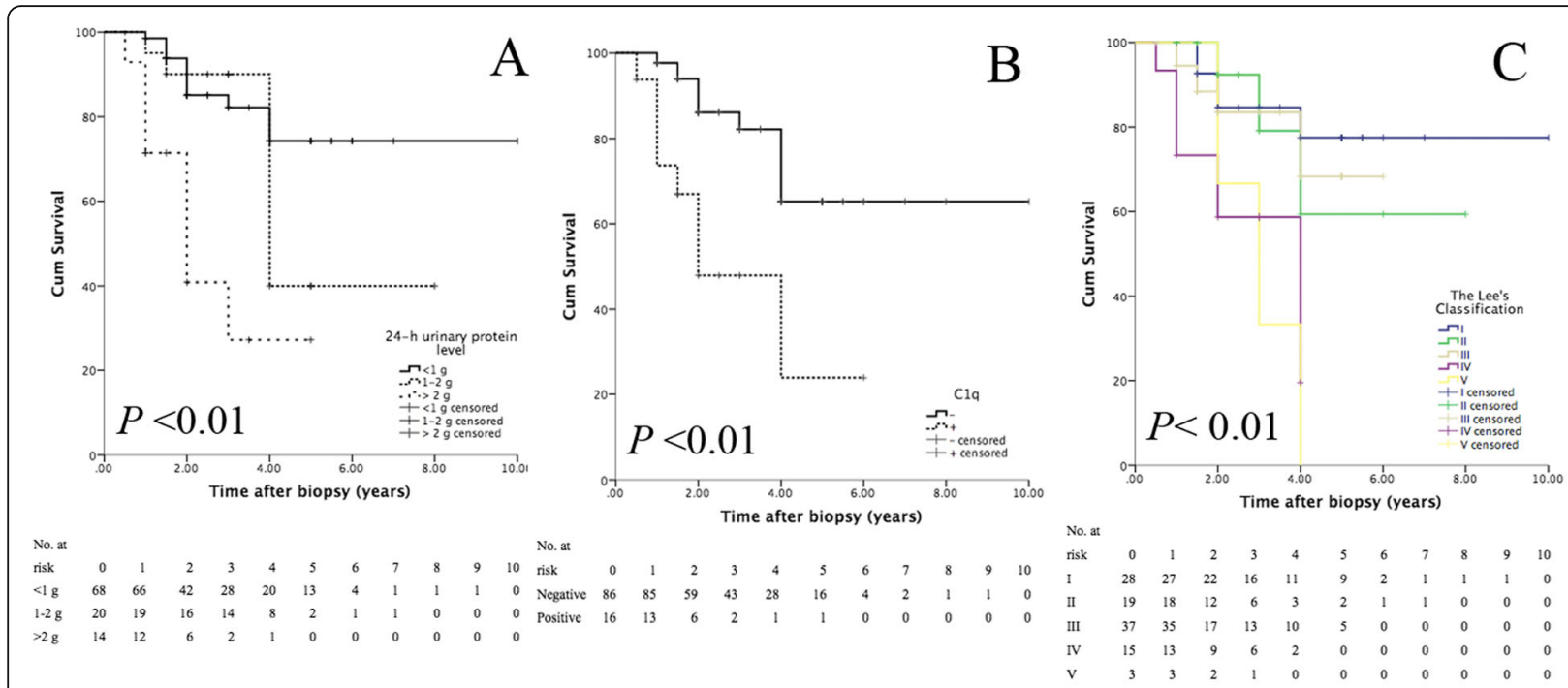

Fig. 3 Kaplan-Meier curves of the 24-h urinary protein level (a), mesangial C1q deposition (b) and Lee's classification (c)

taken seriously when $\mathrm{C} 1 \mathrm{q}$ staining presents during long-term immunosuppression.

Maintenance therapy with medium/low prednisone is used to relieve IgAN progression in combination with angiotensin-converting enzyme inhibitors (ACEIs) to reduce proteinuria. Early steroid withdrawal is a safe intervention in living donor transplantation,[34, 35] but steroid withdrawal should be handled with caution when choosing maintenance immunosuppressive treatments for patients with a high risk of recurrence. Based on our experience of treatment of native IgAN and related references, $[36,37]$ a maintenance therapy of oral prednisone at a dose of $0.6 \mathrm{mg} / \mathrm{kg}$ (qod) for Chinese patients after a IgAN in renal allografts diagnosis may be worthwhile. One recent stud y[38] suggested that other immunosuppressive therapy did not improve outcomes in patients with native IgAN and exhibited a more limited effect in IgAN in renal allografts, which occurred under existing immunosuppression after transplantation. Moreover, patients with IgAN in renal allografts exhibited favorable outcomes after tonsillectomy, $[39,40]$ but no reliable treatment strategy was proven to cure it.

Our study analyzed the 5-year graft cumulative survival rates after transplantation, but longer-term followup from biopsy is needed to observe more outcome events. In most cases, the primary diseases of the recipients were unknown, only 67 cases have been proven primary IgAN by biopsy for native renal in this series, the differences in clinical and pathological characteristics between de novo and recurrent IgAN couldn't be found in this study. Although major confounding variables were adjusted and our results were consistent with other studies in the literature, bias was unavoidable in the univariate Cox proportional hazards models due to the influence of the lack of information for the intraoperative and donor factors.

\section{Conclusion}

IgAN is the most common de novo or recurrent nephropathy, especially in living donor transplantation, and occurs frequently within 5 years after transplantation. The color doppler ultrasound and blood flow imagine were valuable in diagnosing and evaluating the graft dysfunction. The risk of graft failure should be taken seriously in patients who exhibit heavy proteinuria (24-h urinary protein level $>2 \mathrm{~g}$ ) and/or a declined eGFR as the initial symptoms, especially with hypoproteinemia, a high lesion grade (grade IV-V of Lee's classification) and/or mesangial C1q deposition.

\section{Abbreviations}

ACEls: Angiotensin-converting enzyme inhibitors; ALB: Albumin; ARA: Arcuate renal artery; BKVAN: BK virus-associated nephropathy; Cl: Confidence interval; CR: Chronic rejection; CNI: Calcineurin inhibitor; DSAs: Donor-specific antibodies; HRs: Hazard ratios; IgAN: IgA nephropathy; IRA: Interlobar renal artery; KDIGO: Kidney Disease: Improving Global Outcomes; LDL: Low-density lipoprotein; LN: Lupus nephritis; MMF: Mycophenolate mofetil; MRA: Main renal artery; PRA: Panel-reactive antibody; Rl: Resistance index; sCr: Serum creatinine; SRA: Segmental renal atery; U-RBC: Red blood cell; Vs: Peak systolic velocity

\section{Acknowledgements}

The authors would like to thank colleagues in the department of organ transplant, the First Affiliated Hospital of Sun Yat-sen University and the department of urology, Guangzhou Women and Children's Medical Center, for their support and help during this study.

\section{Authors' contributions}

All authors have contributed significant intellectual content to this manuscript as follows: JZ, GDC, JQ, GCL and LZC participated in research design. JZ, GDC, KF and ZXW participated in the literature searching and screening. KF and ZXW participated in the follow-up recording. JZ and GDC participated in the writing of the paper. JZ and GDC participated in data analysis and writing. All authors read and approved the manuscript. 


\section{Funding}

The study was supported by a grant from the National Natural Science Foundation of China (NSFC), which is a government fund used to develop natural science (No. 81470976), and the fund from Guangzhou Institute of Pediatrics/Guangzhou Women and Children's Medical Center (YIP-2019-007). The funding body played no role in the design of the study and collection, analysis, and interpretation of data and in writing the manuscript.

\section{Availability of data and materials}

All data and materials were collected from clinical and pathological database of the First Affiliated Hospital of Sun Yat-sen University and Guangzhou Women and Children's Medical Center. The datasets used and/or analyzed during the current study are available from the author Dr. Jin Zhang (E-mail: m18205188630@163.com) on reasonable request.

\section{Ethics approval and consent to participate}

The Regional Ethics Committee of our center (the First Affiliated Hospital of Sun Yat-sen University and Guangzhou Women and Children's Medical Center) approved this study, and all patients signed informed consent forms.

\section{Consent for publication}

Not applicable.

\section{Competing interests}

The authors declare that they have no competing interests.

\section{Author details}

'Department of Urology, Guangzhou Women and Children's Medical Center, Guangzhou 510000, China. 'Department of Organ Transplant, the First Affiliated Hospital of Sun Yat-sen University, Guangzhou 510000, China.

Received: 27 July 2019 Accepted: 18 November 2019 Published online: 03 December 2019

\section{References}

1. Williams WW, Taheri D, Tolkoff-Rubin N, et al. Clinical role of the renal transplant biopsy. Nat Rev Nephrol. 2012;8(2):110-21.

2. Zhang J, Qiu J, Chen GD, et al. Etiological analysis of graft dysfunction following living kidney transplantation: a report of 366 biopsies. Ren Fail. 2018. https://doi.org/10.1080/0886022X.2018.1455592.

3. El-Zoghby ZM, Stegall MD, Lager DJ, et al. Identifying specific causes of kidney allograft loss. Am J Transplant. 2009;9(3):527-35.

4. Briganti EM, Russ GR, MCNeil JJ, et al. Risk of renal allograft loss from recurrent glomerulonephritis. N Engl J Med. 2002;347(2):103-9.

5. Ponticelli C, Glassock RJ. Posttransplant recurrence of primary glomerulonephritis. Clin J Am Soc Nephrol. 2010;5(12):2363-72.

6. McDonald SP, Russ GR. Recurrence of IgA nephropathy among renal allograft recipients from living donors is greater among those with zero HLA mismatches. Transplantation. 2006;82(6):759-62.

7. Moroni G, Longhi S, Quaglini S, et al. The long-term outcome of renal transplantation of IgA nephropathy and the impact of recurrence on graft survival. Nephrol Dial Transplant. 2013;28(5):1305-14.

8. Kim SJ, Koo HM, Lim BJ, et al. Decreased circulating C3 levels and mesangial C3 deposition predict renal outcome in patients with IgA nephropathy. PLoS One. 2012;7(7):e40495.

9. Lv J, Shi S, Xu D, et al. Evaluation of the Oxford classification of IgA nephropathy: a systematic review and meta-analysis. Am J Kidney Dis. 2013; 62(5):891-9.

10. Floege J, Gröne HJ. Recurrent IgA nephropathy in the renal allograft: not a benign condition. Nephrol Dial Transplant. 2013;28(5):1070-3.

11. Berger J. Recurrence of IgA nephropathy in renal allografts[J]. Am J Kidney Dis. 1988;12(5):371-2.

12. Kidney Disease: Improving Global Outcomes (KDIGO) Transplant Work Group. KDIGO clinical practice guideline for the care of kidney transplant recipients. Am J Transplant. 2009;9(3):S1-155.

13. Kidney disease: Improving Global Outcomes (KDIGO) Glomerulonephritis Work Group. KDIGO clinical practice guideline for glomerulonephritis. Kidney Int. 2012;2:139-274.

14. Foundation NK. K/DOQI clinical practice guidelines for chronic kidney disease: evaluation, classification, and stratification. Am J Kidney Dis. 2002; 39(Suppl1):S1-266
15. Lai KN. Pathogenesis of IgA nephropathy. Nat Rev Nephrol. 2012;8(5):275-83.

16. Nijim S, Vujjini V, Alasfar S, et al. Recurrent IgA nephropathy after kidney transplantation. Transplant Proc. 2016;48(8):2689-94.

17. Floege J. Recurrent IgA nephropathy after renal transplantation. Semin Nephrol. 2004;24(3):287-91.

18. Avasare RS, Rosenstiel PE, Zaky ZS, et al. Predicting post-transplant recurrence of lgA nephropathy: the importance of crescents. Am J Nephrol. 2017;45(2):99-106.

19. Choy BY, Chan TM, Lo SK, et al. Renal transplantation in patients with primary immunoglobulin a nephropathy. Nephrol Dial Transplant. 2003; 18(11):2399-404.

20. Ortiz F, Gelpi R, Koskinen $P$, et al. IgA nephropathy recurs early in the graft when assessed by protocol biopsy. Nephrol Dial Transplant. 2012;27(6):2553-8.

21. Radford MG, Donadio JV, Bergstralh EJ, et al. Predicting renal outcome in IgA nephropathy. J Am Soc Nephrol. 1997;8(2):199-207.

22. Goto M, Wakai K, Kawamura T, et al. A scoring system to predict renal outcome in IgA nephropathy: a nationwide 10-year prospective cohort study. Nephrol Dial Transplant. 2009;24(10):3068-74.

23. Berthoux F, Mohey $\mathrm{H}$, Laurent $\mathrm{B}$, et al. Predicting the risk for dialysis or death in IgA nephropathy. J Am Soc Nephrol. 2011;22(4):752-61.

24. Shu D, Xu F, Su Z, et al. Risk factors of progressive IgA nephropathy which progress to end stage renal disease within ten years: a case-control study. BMC Nephrol. 2017:18(1):11.

25. Platt JF, Rubin JM, Ellis JH. Distinction between obstructive and nonobstructive pyelocaliectasis with duplex Doppler sonography. AJR. 1989; 153(5):997-1000

26. Lee SM, Rao VM, Franklin WA, et al. IgA nephropathy: morphologic predictors of progressive renal disease. Hum Pathol. 1982;13(4):314-22.

27. Hisano S, Joh K, Katafuchi R, et al. Reproducibility for pathological prognostic parameters of the Oxford classification of IgA nephropathy: a Japanese cohort study of the Ministry of Health, labor and welfare. Clin Exp Nephrol. 2017;21(1):92-6.

28. Barbour SJ, Espino-Hernandez G, Reich HN, et al. The MEST score provides earlier risk prediction in IgA nephropathy. Kidney Int. 2016;89(1):167-75.

29. Lee H, Yi SH, Seo MS, et al. Validation of the Oxford classification of IgA nephropathy: a single-center study in Korean adults. Korean J Intern Med. 2012;27(3):293-300.

30. Tanaka S, Ninomiya T, Katafuchi $R$, et al. Development and validation of a prediction rule using the Oxford classification in IgA nephropathy. Clin J Am Soc Nephrol. 2013;8(12):2082-90.

31. Trimarchi H, Barratt J, Cattran DC, et al. Oxford classification of IgA nephropathy 2016-the role of crescentic lesions: an update from the IgA nephropathy classification working group. Kidney Int. 2017;91(5):1014.

32. Lee HJ, Choi SY, Jeong KH, et al. Association of C1q deposition with renal outcomes in IgA nephropathy. Clin Nephrol. 2013;80(2):98-104.

33. Nishiwaki $H$, Hasegawa $T$, Nagayama $Y$, et al. Absence of mesangial C1q deposition is associated with resolution of proteinuria and hematuria after tonsillectomy plus steroid pulse therapy for immunoglobulin a nephropathy. Nephron. 2015;130(1):1-7.

34. Andrade-Sierra J, Rojas-Campos E, Cardona-Muñoz E, et al. Early steroid withdrawal in recipients of a kidney transplant from a living donor: experience of a single Mexican center. Transplant Proc. 2016;48(1):42-9.

35. Cantarovich D, Rostaing L, Kamar N, et al. Early corticosteroid avoidance in kidney transplant recipients receiving ATG-F induction: 5-year actual results of a prospective and randomized study. Am J Transplant. 2014;14(11):2556-64.

36. Ponticelli C, Glassock RJ. IgA nephritis with declining renal function: treatment with corticosteroids may be worthwhile. J Am Soc Nephrol. 2015;26(9):2071-3.

37. Pozzi C, Andrulli S, Del Vecchio L, et al. Corticosteroid effectiveness in IgA nephropathy: long-termresults of a randomized, controlled trial. J Am Soc Nephrol. 2004;15(1):157-63.

38. Rauen T, Eitner F, Fitzner $C$, et al. Intensive supportive care plus immunosuppression in lgAnephropathy. N Engl J Med. 2015;373(23):2225-36.

39. Hotta K, Fukasawa Y, Akimoto M, et al. Tonsillectomy ameliorates histological damage of recurrent immunoglobulin a nephropathy after kidney transplantation. Nephrology. 2013;18(12):808-12.

40. Ushigome $H$, Suzuki T, Fujiki M, et al. Efficacy of tonsillectomy for patients with recurrence of IgA nephropathy after kidney transplantation. Clin Transpl. 2009;23(S20):17-22.

\section{Publisher's Note}

Springer Nature remains neutral with regard to jurisdictional claims in published maps and institutional affiliations. 